\title{
International variations and trends in the treatment for retinopathy of prematurity
}

\author{
Brian A Darlow, ${ }^{1}$ Kei Lui, ${ }^{2}$ Satoshi Kusuda, ${ }^{3}$ Brian Reichman, ${ }^{4}$ Stellan Håkansson, ${ }^{5}$ \\ Dirk Bassler, ${ }^{6}$ Neena Modi, ${ }^{7}$ Shoo K Lee, ${ }^{8,9}$ Liisa Lehtonen, ${ }^{10}$ Maximo Vento, ${ }^{11}$ \\ Tetsuya Isayama, ${ }^{8}$ Gunnar Sjörs, ${ }^{12}$ Kjell K Helenius, ${ }^{10}$ Mark Adams, ${ }^{6}$ \\ Franca Rusconi, ${ }^{13,14}$ Naho Morisaki, ${ }^{15}$ Prakesh S Shah,,${ }^{8,9}$ \\ on behalf of the International Network for Evaluating Outcomes of Neonates
}

\begin{abstract}
- Additional material is published online only. To view please visit the journal online (http://dx.doi.org/10.1136/ bjophthalmol-2016-310041).
\end{abstract}

For numbered affiliations see end of article.

Correspondence to Professor Brian A Darlow, Department of Paediatrics, University of Otago, PO Box 4345, Christchurch 8140, New Zealand;

brian.darlow@otago.ac.nz

Received 9 December 2016 Revised 19 January 2017 Accepted 10 February 2017 Published Online First 7 March 2017
CrossMark

To cite: Darlow BA, Lui K, Kusuda $\mathrm{S}$, et al.

Br J Ophthalmol

2017;101:1399-1404.

\section{ABSTRACT}

Objective To compare the rates of retinopathy of prematurity (ROP) and treatment of ROP by laser or intravitreal anti-vascular endothelial growth factor among preterm neonates from high-income countries

participating in the International Network for Evaluating Outcomes (iNeo) of neonates.

Methods A retrospective cohort study was conducted on extremely preterm infants weighing $<1500 \mathrm{~g}$ at $24^{0}$ to $27^{6}$ weeks' gestation who were admitted to neonatal units in Australia/New Zealand, Canada, Finland, Israel, Japan, Spain, Sweden, Switzerland, Tuscany (Italy) and the UK between 2007 and 2013. Pairwise comparisons of ROP treatment in survivors between countries were evaluated by Poisson and multivariable logistic regression analyses after adjustment for confounders. A composite outcome of death or ROP treatment was compared between countries using logistic regression and standardised ratios.

Results Of 48087 infants included in the analysis, $81.8 \%$ survived to 32 weeks postmenstrual age, and $95 \%$ of survivors were screened for ROP. Rates of any ROP ranged from $25.2 \%$ to $91.0 \%$ in Switzerland and Japan, respectively, among those examined. The overall rate of those receiving treatment was $24.9 \%$, which varied from $4.3 \%$ to $30.4 \%$. Adjusted risk ratios for ROP treatment were lower for Switzerland in all pairwise comparisons, whereas Japan displayed significantly higher ratios. Comparisons of the composite outcome between countries revealed similar, but less marked differences.

Conclusions Rates of any ROP and ROP treatment varied significantly between iNeo members, while an overall decline in ROP treatment was observed during the study period. It is unclear whether these variations represent differences in care practices, diagnosis and/or treatment thresholds.

\section{INTRODUCTION}

Retinopathy of prematurity (ROP), a disease characterised by abnormal blood vessel growth in the retina, is a significant complication of very preterm birth in high-income countries, and increasingly so in low/middle-income countries. ${ }^{1}$ From early descriptions of the disease to the modern day, the incidence and treatment of ROP has varied considerably between hospitals. ${ }^{2-5}$ The reasons for such variation are complex and may include differences in case-mix, case ascertainment, treatment thresholds, hospital size and variations in clinical care, among others. ${ }^{2-4} 67$ Understanding the extent and causes of variation at both the local and international level may help inform future care and provide greater consistency in diagnosis and treatment thresholds.

The past two decades have witnessed a rise in national neonatal networks, which undertake ongoing audits of high-risk infants and, mainly through collaborative quality improvement efforts, have led to advances in neonatal care and better overall outcomes. ${ }^{2}{ }^{6}$ Recognising the need for international comparisons of neonatal morbidity rates, the International Network for Evaluating Outcomes (iNeo) in Neonates was formed. ${ }^{7}$ The iNeo comprises 10 population-based national neonatal networks from 11 countries: Australia and New Zealand(ANZNN), Canada (CNN), Israel (INN), Japan (NRNJ), Sweden (SNQ), Switzerland (SwissNeoNet), Spain (SEN1500), the UK Neonatal Collaborative (UKNC), Finland (FinMBR) and Tuscany (TuscanNN). Here, our objective was to compare ROP incidence and treatment (by laser or intravitreal anti-vascular endothelial growth factor (anti-VEGF)) in very preterm neonates between networks in the iNeo consortium.

\section{METHODS}

\section{Study design and population}

This study included a retrospective comparison of data on neonates born between $24^{0}$ and $27^{6}$ weeks gestational age (GA) with birth weights $<1500 \mathrm{~g}$ and admitted to a neonatal unit in a collaborating network between 2007 and 2013. Infants with major congenital anomalies were excluded, as well as extremely preterm infants $<24$ weeks, at which gestation neonatal intensive care provision varies considerably among iNeo networks. We included only neonates of $24^{0}-27^{6}$ weeks GA because all surviving infants at this gestation are screened. Approvals for data collection and data sharing agreements were obtained by individual networks and the iNeo Coordinating Centre at Mount Sinai Hospital, Toronto, Ontario, Canada.

\section{Outcomes and measures}

Data on outcomes of eye examinations were obtained from all individual network databases. Most eye examinations were performed by fundoscopy and very few babies during the study period had wide-field retinal photography. All networks 
recorded data using the International Classification of ROP. The highest stage of ROP was recorded, if present, along with any treatment for advanced stages of ROP by laser or by intravitreal anti-VEGF, but data on timing of the initial examination, frequency of examinations, zone, extent of disease (clock hours) and presence or absence of plus disease were not captured. Information on mortality prior to 32 weeks postmenstrual age (PMA) was obtained and to account for differences in mortality between networks, a composite outcome of death or treated ROP was analysed.

\section{Statistical analysis}

Rates of any ROP, stage 3 or higher ROP and treatment in surviving infants examined for ROP were calculated for each network. Data from the entire cohort were evaluated by Poisson and multivariable logistic regression models to determine risk factors for ROP treatment. Risk ratios (RRs) with 95\% CIs were reported. Multivariable logistic regression and Poisson regression analyses were applied to compare ROP treatment among all networks simultaneously with adjustment for GA, birth weight z-score, multiple birth, sex, caesarean section (CS) and antenatal steroids. Adjusted RRs were estimated for all possible pairwise comparisons. Statistical significance was evaluated by applying stringent Bonferroni multiple-testing adjustments to account for 45 independent pairwise comparisons with a significance threshold of $\mathrm{p}<0.001$ (corresponding to $99.9 \%$ CI for $\mathrm{RR}$ estimates). Standardised ratios (SRs) were calculated as the observed number of infants who received ROP treatment divided by the number of infants expected to receive ROP treatment, computed as the sum of predicted probabilities from a multivariable logistic regression model derived using data from all other countries. As the SR estimate is calculated in relation to all other countries combined, it is not directly comparable between contributors. The Cochran-Armitage trend test was used to assess the trend rates of ROP treatment by admission year for each network for the whole study population, as well as for various GA groups. Data management and statistical analyses were performed at the iNeo Coordinating Centre using SAS V.9.2 (SAS Institute).

\section{RESULTS}

A total of 48087 infants of $24^{0}-27^{6}$ weeks gestation were included in the analysis. Mean survival to at least 32 weeks PMA was $81.8 \%$ and varied from $91.8 \%$ in Japan to $67.8 \%$ in Spain (table 1). There was a higher proportion of the most immature (24 weeks GA) and extremely low birth weight $(<1000 \mathrm{~g})$ infants among survivors in Japan compared with other networks. Results of ROP screening in infants alive at 32 weeks PMA were available for $95.0 \%$ of eligible infants, with missing data varying between $0.4 \%$ and $11.4 \%$ (table 1 ). Among the infants examined for ROP, any ROP rates ranged from $25.2 \%$ to $91.0 \%$, and treatment varied between $4.3 \%$ and $30.4 \%$. The composite outcome of death or ROP treatment varied between $22.1 \%$ and $40.6 \%$ (table 1 ).

Analysis of the full dataset indicated that non-receipt of antenatal steroids, male sex, lower GA, lower birth weight for gestation and delivery by CS were associated with ROP treatment (table 2), whereas multiple births was not.

Table 3 highlights relative risk comparisons between pairs of networks for ROP treatment in surviving infants examined for ROP. Switzerland had a lower risk of ROP treatment in all pairwise comparisons with other countries. Similar results were obtained when caesarean delivery and antenatal steroids were not adjusted for (see online supplementary table S1). However,

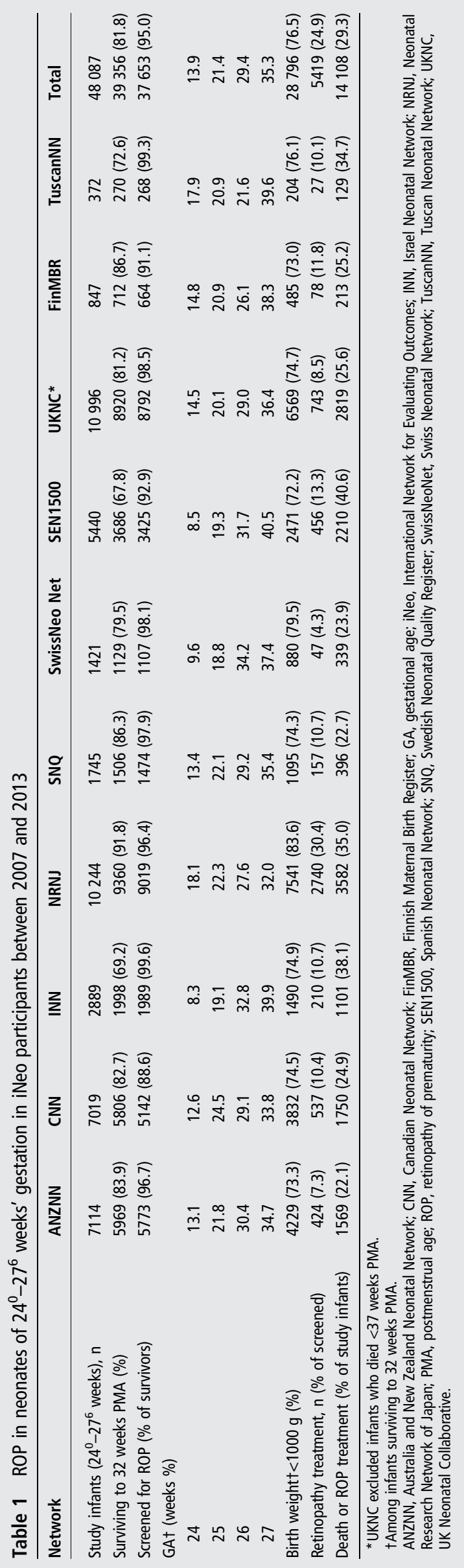


Table 2 Factors associated with ROP treatment

\begin{tabular}{ll}
\hline Risk factor & RR $(95 \% \mathrm{Cl})^{*}$ \\
\hline $\begin{array}{l}\text { No antenatal steroids } \\
\text { Male sex }\end{array}$ & $1.66(1.57$ to 1.75$)$ \\
GA & $1.08(1.03$ to 1.13$)$ \\
24 vs 27 & \\
25 vs 27 & $5.23(4.83$ to 5.65$)$ \\
26 vs 27 & $3.46(3.20$ to 3.74$)$ \\
BWZ score (per 1 unit increase-equivalent to 1 SD & $1.89(1.74$ to 2.05$)$ \\
increase) & $0.76(0.74$ to 0.78$)$ \\
Caesarean section & \\
Multiples & $1.26(1.19$ to 1.33$)$ \\
\hline
\end{tabular}

*Derived by Poisson analyses.

BWZ, birth weight z-score; GA, gestational age; ROP, retinopathy of prematurity; RR, risk ratio.

when death or ROP treatment was used as the outcome, Switzerland did not differ from five of the networks (see online supplementary table S2). Japan had a higher relative risk for any ROP (data not shown) and ROP treatment in comparison to all other iNeo countries.

A comparison of estimated SRs of the composite outcome of each country relative to one another is graphically displayed in figure 1. Figure $1 \mathrm{~A}$ reveals that the SR for Japan was significantly higher, whereas Switzerland, Australia/New Zealand and the UK were significantly lower. Figure $1 \mathrm{~B}$ illustrates the same data excluding Japan. In this analysis, the estimated SR for Spain was significantly higher compared with all others, whereas the SR for Switzerland and the UK remained significantly lower.

The rate of ROP treatment declined significantly from 2007 to 2013 within the overall collaboration, although the trend from 2008 to 2013 was stable. The trend in the latter period also remained constant in most individual networks, with the exception of the UK, where the rate of treatment increased significantly (table 4). In the full 7 -year period, there was an overall significant decline in rates of treatment for infants of 26-27 weeks' gestation, but not at $<26$ weeks GA (see online supplementary table S3).

\section{DISCUSSION}

We have identified considerable variation in rates of treatment for ROP between countries after adjustment for risk factors. The strengths of our study include the very large sample size and ability to report on temporal trends. Weaknesses include the fact that this is a retrospective analysis of a minimum dataset. Possible explanations for the variation detected include differences in network coverage of the relevant population, differences in population and ethnic and genetic characteristics, and variations in care practices and treatment thresholds. The size of individual networks may also be a contributor, as smaller networks tend to display greater intrinsic variability due to rarity of the event of interest. To reduce bias from differences in population coverage, we only included neonates of $24^{0}-27^{6}$ weeks GA, when screening is standard in all networks. Some missing data may result from back transfer of infants to a lower level neonatal unit from which data might not have been captured by most countries other than the UK.

Consistent with earlier findings, we demonstrated that receipt of antenatal corticosteroids is associated with a reduced risk of ROP treatment, ${ }^{9}{ }^{10}$ while lower GA and lower birth weight for gestation are important risk factors. ${ }^{11} 12$ Our data also suggest

గฺุ 广 5 厂

z

อ อ

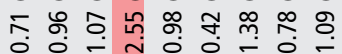

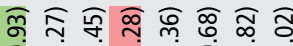

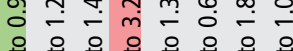

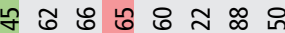

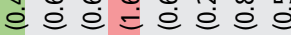

గొ

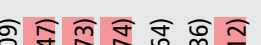

它舀

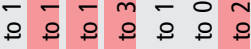

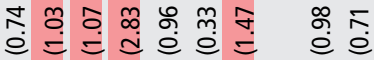

兰

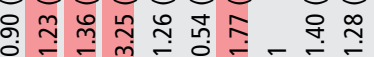

గิ犬 శু

๒ั

क̊

요원

8

于

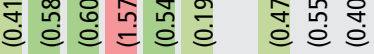

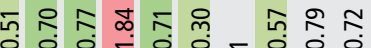

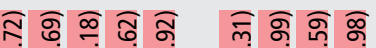

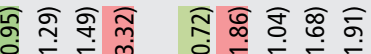

웅

ㅊํㅇ \% 웅 응 

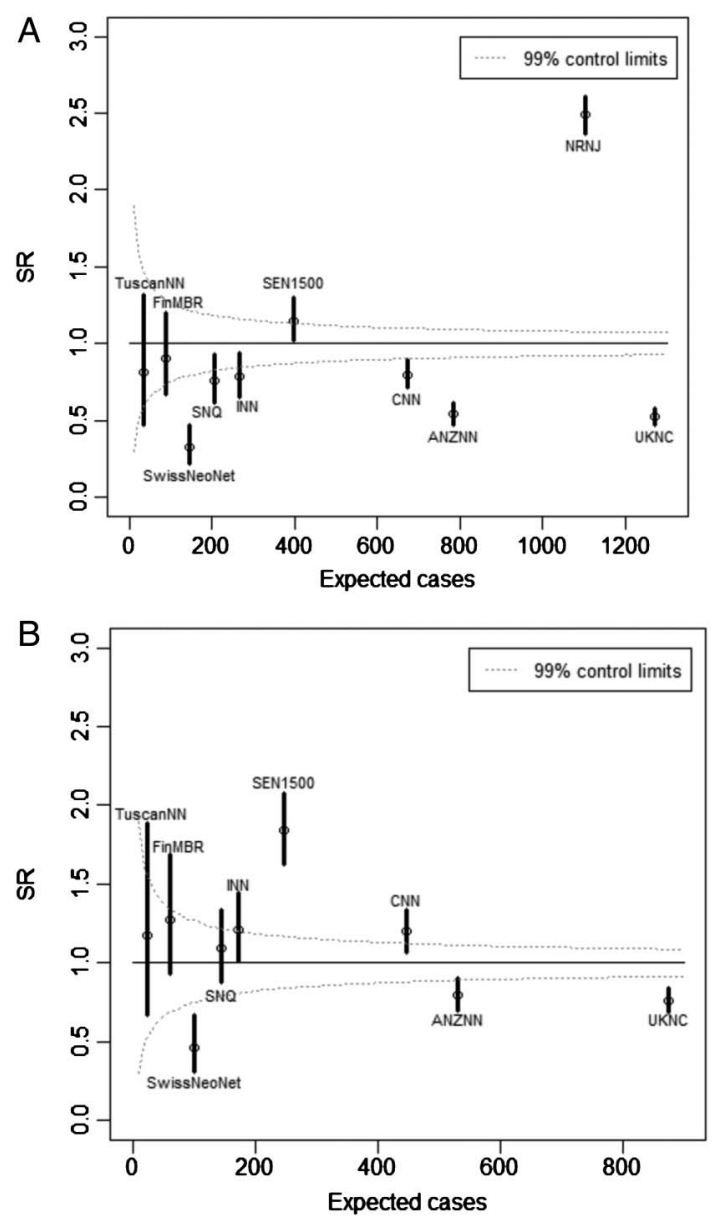

Figure 1 Standardised ratios (SRs) of the composite outcome. ${ }^{a}$ (A) Estimated SRs (and 99\% Cls) of the composite outcome of all networks included in iNeo (calculated using logistic regression analyses); (B) estimated SRs (and $99 \% \mathrm{Cls}$ ) of the composite outcome of all iNeo members, excluding Japan (calculated using logistic regression analyses). ${ }^{\text {a }}$ SRs comparing the composite outcome of each network with all other networks combined. Vertical bars are the estimated $99 \%$ $\mathrm{Cls}$ of the SR. The dotted curves represent the $99 \%$ control limits expected under the null hypothesis of similar outcome rates $(S R=1)$. ANZNN, Australian and New Zealand Neonatal Network; CNN, Canadian Neonatal Network; FinMBR, Finnish Maternal Birth Register; iNeo, International Network for Evaluating Outcomes; INN, Israel Neonatal Network; NRNJ, Neonatal Research Network of Japan; SEN1500, Spanish Neonatal Network; SNQ, Swedish Neonatal Quality Register; SwissNeoNet, Swiss Neonatal Network; TuscanNN, Tuscan Neonatal Network; UKNC, UK Neonatal Collaborative.

that males have an increased risk of ROP, similar to other studies. $^{5} 111^{13}$ In contrast, delivery by CS, which has become more frequent for very preterm infants during this time period, ${ }^{14}$ has not been previously reported as a risk factor for severe ROP. The Neonatal Research Network of Japan ${ }^{10}$ reported a significant reduction in the risk of $\geq$ stage 3 ROP for infants delivered by CS compared with vaginal delivery. The relationship between mode of delivery and severe ROP is likely to be complex and warrants further analysis.

Most iNeo countries, with the exception of Japan, are ethnically diverse and this may also contribute to our observed variation. A lower risk of ROP was reported among AfricanAmerican infants in comparison with Caucasian infants, ${ }^{15}$ whereas Hispanic ${ }^{16}$ and Asian infants from the Indian subcontinent ${ }^{17}$ display an increased risk. A study of two large cohorts from the USA revealed that a single nucleotide polymorphism in the brain-derived neurotrophic factor gene was associated with severe (threshold) ROP. ${ }^{18}$

Survival also impacts ROP rates, as very sick infants may not survive long enough to receive a retinal examination. When we analysed the combined outcome of death or ROP treatment, some differences between networks were no longer apparent. In high-income countries, including iNeo members, improved survival through better care practices has been frequently accompanied by stable or lower morbidity rates. ${ }^{14} 19$ The overall trend in our dataset was a decline in treatment rates from 2007 to 2013, but stable in the last 6 years. A population-based report from Northern Ireland noted increased rates of treatment for ROP from 2000 to 2011, and indicated that earlier treatment of ROP (ET-ROP) criteria ${ }^{20}$ was not incorporated into UK guidelines until 2008. ${ }^{21} \mathrm{~A}$ review of hospital data from England for the years 1990-2011 also reported an increase in the rates of treatment for ROP between 2005 and $2011 .^{22}$ The UKNC has reported an annual increase in the proportion of infants meeting ROP screening criteria being examined on time; hence, the rise in treatment rates may reflect improved ascertainment. ${ }^{23}$

In our study, Japan had the highest survival rate at 92\%, and experienced a much higher ROP treatment rate compared with all other networks. A previous study comparing the $\mathrm{CNN}$ and NRNJ between 2006 and 2008 revealed that Japanese infants had higher rates of severe ROP. ${ }^{24}$ In Japan, the mean number of days of ventilation and oxygen treatment was higher than in Canada, as was the rate of bronchopulmonary dysplasia, which may have contributed to the increased risk of ROP. ${ }^{24}$ However, another possibility is that diagnosis and treatment criteria for ROP are different in Japan.

The Cryotherapy for ROP (CRYO-ROP) study ${ }^{25}$ established the treatment criteria of 'threshold disease' (five contiguous or eight total clock hours of stage 3 with plus disease), and this was widely used until the Early Treatment for ROP (ETROP) trial showed improved outcomes with earlier treatment at 'type 1' ROP (zone I, any ROP with plus disease; zone I, any stage 3 ROP; zone II, stage 2 or 3 with plus disease). ${ }^{20}$ Preliminary results from our survey on current treatment practices in 10 of the 11 iNeo networks, including criteria for ROP treatment, show that $65 \%$ of neonatal intensive care units use ET-ROP type 1 criteria, $27 \%$ use the threshold and $7 \%$ use other criteria. All networks showed variation in treatment criteria and we are currently exploring this in more detail together with other practices, including oxygen saturation targets.

In our analysis, the number of infants in Japan who were treated for ROP exceeded those documented as having stage 3 ROP by $8.1 \%$. In all other countries, except Spain and Finland, where treatment exceeded stage 3 ROP by $2.7 \%$ and $0.7 \%$, respectively, fewer infants were treated than had stage 3 ROP. The Japanese classification of ROP is slightly different from the international classification, ${ }^{8}$ and a subset of infants who would normally be classified with stage 2 disease would be considered stage '3 early' (see online supplementary table S4). Additionally, given the fear of litigation, it is likely that some ophthalmologists in Japan treat infants at an earlier stage than type I ROP (S. Kusuda, personal communication, 2016). Equally, in Spain and Finland it is possible that infants with 'imminent' stage 3 were treated. A major determinant of treatment using ET-ROP criteria is the presence of 'plus' disease, which is not collected by most networks.

Ascertainment bias, including subjective interpretation of the retina as seen with the indirect ophthalmoscope, ${ }^{26}$ may be a contributing factor to the variation in severe ROP incidence rates. In the CRYO-ROP study, experts disagreed on the 
Table 4 ROP treatment by year in individual participants for the entire cohort ( $<28$ weeks GA)

\begin{tabular}{lcccccccrrr}
\hline Network & 2007 & 2008 & 2009 & 2010 & 2011 & 2012 & 2013 & $p$ Trend & $p$ Trend excluding 2007 \\
\hline ANZNN & $71(8.2)$ & $72(8.3)$ & $58(7.4)$ & $46(6.1)$ & $64(7.8)$ & $57(6.9)$ & $56(6.6)$ & 0.13 & 0.26 \\
CNN & $88(14.8)$ & $86(13.2)$ & $70(10.8)$ & $91(11.6)$ & $71(9.8)$ & $67(8.8)$ & $64(8.9)$ & $<0.01$ & $<0.01$ \\
INN & $38(13.9)$ & $35(13.0)$ & $33(11.4)$ & $24(7.8)$ & $31(10.7)$ & $24(9.3)$ & $25(8.3)$ & 0.01 & 0.08 & 0.01 \\
NRNJ & $431(36.4)$ & $311(26.2)$ & $381(30.5)$ & $424(30.7)$ & $402(29.5)$ & $439(31.4)$ & $352(28.0)$ & 0.03 & 0.35 & 0.05 \\
SNQ & $26(12.8)$ & $21(11.3)$ & $23(11.1)$ & $30(13.9)$ & $25(13.5)$ & $18(7.9)$ & $17(6.6)$ & 0.02 & 0.05 \\
SwissNeoNet & $5(4.0)$ & $7(4.7)$ & $6(4.2)$ & $12(7.3)$ & $5(2.8)$ & $6(3.1)$ & $6(3.9)$ & 0.49 & 0.34 & 0.83 \\
SEN-1500 & $65(14.7)$ & $54(11.5)$ & $64(12.5)$ & $77(15.8)$ & $65(13.3)$ & $66(12.4)$ & $65(13.2)$ & 0.83 & 0.68 \\
UKNC & $*$ & $59(5.2)$ & $61(5.5)$ & $138(9.2)$ & $148(8.8)$ & $142(8.3)$ & $195(11.9)$ & $<0.01$ & $<0.01$ \\
FinMBR & $11(12.1)$ & $8(7.6)$ & $19(20.7)$ & $8(9.1)$ & $14(12.3)$ & $11(11.5)$ & $7(9.1)$ & 0.70 & 0.68 \\
TuscanNN & $*$ & $*$ & $8(13.3)$ & $4(8.2)$ & $5(10.2)$ & $4(7.7)$ & $6(10.3)$ & 0.59 & 0.59 \\
Total & $735(19.4)$ & $653(13.0)$ & $723(14.2)$ & $854(14.9)$ & $830(14.1)$ & $834(13.7)$ & $793(13.7)$ & $<0.01$ & 0.95 \\
\hline
\end{tabular}

*Data not available.

ANZNN, Australia and New Zealand Neonatal Network; CNN, Canadian Neonatal Network; FinMBR, Finnish Maternal Birth Register; GA, gestational age; INN, Israel Neonatal Network; NRNJ, Neonatal Research Network of Japan; ROP, retinopathy of prematurity; SEN1500, Spanish Neonatal Network; SNQ, Swedish Neonatal Quality Register; SwissNeoNet, Swiss Neonatal Network; TuscanNN, Tuscan Neonatal Network; UKNC, UK Neonatal Collaborative.

presence or absence of threshold disease for $12 \%$ of the cases, particularly regarding the presence or absence of plus disease. ${ }^{27}$ While standard retinal examinations are performed by indirect ophthalmoscopy, digital imaging is being increasingly employed, either at the same time (to serve as a permanent record) or alone (to be interpreted locally or remotely). ${ }^{28}$ Nonetheless, digital photographs still require interpretation and may be subject to bias.

Differences in clinical practices could also have a major impact on rates of ROP severe enough to require treatment. Nosocomial sepsis, inadequate nutrition and poor growth, prolonged supplementary oxygen and chronic neonatal lung disease have all been identified as risk factors for ROP and all potentially can be decreased through the implementation of evidence-based practices. ${ }^{29}$ For most of the study years, there were no nationally adopted recommendations either for initial inspired oxygen concentrations at neonatal resuscitation in infants $<28$ weeks' gestation or for oxygen saturation targets in the neonatal intensive care unit, although the latter were typically within the range of $85 \%-95 \%{ }^{30}$ Recent trials of oxygen saturation targeting indicated that infants cared for with a target at the upper end of this range (91\%-95\%) experienced both higher survival and higher rates of severe ROP compared with infants cared for at the lower end $(85 \%-89 \%) .{ }^{31}$ It will be important to monitor future trends in both survival and ROP as neonatologists reassess appropriate oxygen saturation targets for these high-risk patients.

We have reported considerable variation in the rates of any ROP and ROP treatment among the iNeo collaboration, although there was less variation in the composite outcome of death or ROP treatment. Differences in ascertainment of ROP and ROP treatment thresholds may contribute to the variability observed, as well as differences in care provision. Exploring care practices associated with better outcomes should benefit future generations of extremely preterm infants. To understand differences in outcomes, networks should adopt common definitions to describe pathology and improve consistency in interpretation, minimise the amount of missing data and perhaps record an expanded dataset; specifically, for ROP, this should include information on plus disease and aggressive posterior-ROP. ${ }^{32}$ Perhaps the most important outcomes for families of extremely preterm infants beyond survival are long-term visual outcomes. Several iNeo networks now record neurodevelopmental outcomes at 18 months to 3 years and linking neonatal datasets with visual outcomes during childhood will be a major advance.

\section{Author affiliations}

${ }^{1}$ Australia and New Zealand Neonatal Network, Department of Paediatrics, University of Otago, Christchurch, New Zealand

${ }^{2}$ Australian and New Zealand Neonatal Network, Royal Hospital for Women, National Perinatal Epidemiology and Statistic Unit, University of New South Wales, Randwick, Australia

${ }^{3}$ Neonatal Research Network Japan, Maternal and Perinatal Center, Tokyo Women's Medical University, Kawadacho, Shinjuku, Tokyo, Japan

${ }^{4}$ Israel Neonatal Network, Gertner Institute for Epidemiology and Health Policy Research, Sheba Medical Centre, Tel Hashomer, Israel

${ }^{5}$ Swedish Neonatal Quality Register, Department of Pediatrics/Neonatal Services, Umeå University Hospital, Umeå, Sweden

${ }^{6}$ Swiss Neonatal Network, Department of Neonatology, University Hospital Zurich, University of Zurich, Frauenklinikstrasse, Zurich, Switzerland

${ }^{7}$ UK Neonatal Collaborative, Neonatal Data Analysis Unit, Section of Neonatal Medicine, Department of Medicine, Imperial College London, Chelsea and Westminster Hospital campus, London, UK

${ }^{8}$ Department of Paediatrics, Mount Sinai Hospital and University of Toronto, Toronto, Ontario, Canada

${ }^{9}$ Canadian Neonatal Network, Maternal-Infant Care Research Centre, Mount Sinai Hospital, Toronto, Ontario, Canada

${ }^{10}$ Finnish Medical Birth Register and Register of Congenital Malformations, Department of Pediatrics, Turku Univeristy Hospital, Kinamyllynkatu, Turku, Finland

${ }^{11}$ Spanish Neonatal Network, Health Research Institute La Fe, Avenida Fernando Abril Martorell, Valencia, Spain

${ }^{12}$ Swedish Neonatal Quality Register, Department of Women's and Children's Health, Uppsala University, Uppsala, Sweden

${ }^{13}$ TIN Toscane Online, Unit of Epidemiology, Meyer Children's University Hospital, Viale Pieraccini, Florence, Italy

${ }^{14}$ Regional Health Agency, Via Pietro Dazzi, Florence, Italy

${ }^{15}$ Neonatal Research Network Japan, Department of Social Medicine, National

Center for Child Health and Development, Okura, Setagaya, Tokyo, Japan

Acknowledgements The authors gratefully acknowledge all investigators and data abstractors of networks participating in the iNeo consortium for their diligent work. The authors also thank staff at the Maternal-Infant Care Research Centre in Toronto, Ontario, for organisational, statistical and editorial support. The authors acknowledge the contribution of the SWEDROP register (Director Gerd Holmström MD), which is linked to The Swedish Neonatal Quality Register to provide information on ROP and Dr Luigi Gagliardi for his valuable comments on the manuscript.

Collaborators Group Information: Investigators of the International Network for Evaluating Outcomes (iNeo) of Neonates are provided in the online only supplementary material.

Contributors BAD conceptualised and designed the study, contributed to the interpretation of data, drafted the initial manuscript and approved the final manuscript as submitted. KL, SK, BR, SH, DB, NM, SKL, LL, MV, TI, GS, KKH, FR, NM, PSS and MA contributed to the concept, design and interpretation of data, critically reviewed and revised the draft manuscript for intellectual content and approved the final submitted version of the article. All authors agree to be accountable for all aspects of the work presented, including the accuracy and integrity of the findings reported. PSS had full access to all the data in the study and takes responsibility for the integrity of the data and the accuracy of the data analysis. 
Funding Funding for iNeo has been provided by a Canadian Institutes of Health Research Chair in Reproductive and Child Health Services and Policy Research (APR-126340) held by PSS. The Canadian Neonatal Network is funded by financial support from the Canadian Institutes of Health Research (FRN87518) and individual participating centres. The Australian and New Zealand Neonatal Network is predominantly funded by membership contributions from participating centres. The Israel Neonatal Network very low birth weight infant database is partially funded by the Israel Center for Disease Control and the Ministry of Health. TIN Toscane online Network is funded by the Tuscany Region. The Neonatal Research Network of Japan is partly funded by a Health Labour Sciences Research Grant from the Ministry of Health, Labour and Welfare of Japan. SEN1500 is supported by funds from the Spanish Neonatal Society. The Swedish Neonatal Quality Register is funded by the Swedish Government (Ministry of Health and Social Affairs) and the body of regional healthcare providers (County Councils). SwissNeoNet is partially funded by participating units in the form of membership fees. The UK Neonatal Collaborative receives no core funding.

Disclaimer The funding bodies played no role in the design and conduct of the study; collection, management, analysis and interpretation of the data; preparation, review or approval of the manuscript; and decision to submit the manuscript for publication.

Competing interests None declared.

Ethics approval Mount Sinai Hospital.

Provenance and peer review Not commissioned; externally peer reviewed.

\section{REFERENCES}

1 Blencowe $H$, Lawn JE, Vazquez $T$, et al. Preterm-associated visual impairment and estimates of retinopathy of prematurity at regional and global level for 2010 . Pediatr Res 2013;74(Suppl 1):36-49.

2 Lee SK, McMillan DD, Ohlsson A, et al. Variations in practice and outcomes in the Canadian NICU Network: 1996-1997. Pediatrics 2000;106:1070-9.

3 Darlow BA, Hutchinson JL, Simpson JM, et al. Variation in rates of severe retinopathy of prematurity among neonatal intensive care units in the Australian and New Zealand Neonatal Network. Br J Ophthalmol 2005;89:1592-6.

4 Kusuda S, Fujimura M, Sakuma I, et al. Morbidity and mortality of infants with very low birth weight in Japan: center variation. Pediatrics 2006;118:e1130-38.

5 Thomas K, Shah PS, Canning R, et al. Retinopathy of prematurity: risk factors and variability in Canadian neonatal intensive care units. J Neonatal Perinatal Med 2015;8:207-14.

6 Horbar JD, Badger GJ, Lewitt EM, et al. Hospital and patient characteristics associated with variation in 28-day mortality rates for very low birth weight infants. Vermont Oxford Network. Pediatrics 1997;99:149-56.

7 Shah PS, Lee SK, Lui K, et al. The International Network for Evaluating Outcomes of very low birth weight, very preterm neonates (iNeo): a protocol for collaborative comparisons of international health services for quality improvement in neonatal care. BMC Pediatr 2014;14:110.

8 International Committee for the Classification of Retinopathy of Prematurity. The international classification of retinopathy of prematurity revisited. Arch Ophthalmol 2005;123:991-9.

9 Higgins RD, Mendelsohn AL, DeFeo MJ, et al. Antenatal dexamethasone and decreased severity of retinopathy of prematurity. Arch Ophthalmol 1998;116:601-5.

10 Sasaki Y, Ikeda T, Nishimura K, et al. Association of antenatal corticosteroids and the mode of delivery with the mortality and morbidity of infants weighing less than $1,500 \mathrm{~g}$ at birth in Japan. Neonatology 2014;106:81-6.
11 Darlow BA, Hutchinson JL, Henderson-Smart DJ, et al. Prenatal risk factors for severe retinopathy of prematurity among very preterm infants of The Australian and New Zealand Neonatal Network. Pediatrics 2005;115:990-6.

12 Hellström A, Smith LEH, Dammann O. Retinopathy of prematurity. Lancet 2013;382:1445-57.

13 van Sorge AJ, Termote JUM, Kerkhoff FT, et al. Nationwide inventory of risk factors for retinopathy of prematurity in the Netherlands. J Pediatr 2014;164:494-8.e1.

14 Stoll BJ, Hansen NI, Bell EF, et al. Trends in care practices, morbidity, and mortality of extremely preterm neonates, 1993-2012. JAMA 2015;314:1039-51.

15 Saunders RA, Donahue ML, Christmann LM, et al. Racial variation in retinopathy of prematurity. The Cryotherapy for Retinopathy of Prematurity Cooperative Group. Arch Ophthalmol 1997;115:604-8.

16 Lad EM, Nguyen TC, Morton JM, et al. Retinopathy of prematurity in the United States. Br J Ophthalmol 2008;92:320-5.

17 Husain SM, Sinha AK, Bunce C, et al. Relationships between maternal ethnicity, gestational age, birth weight, weight gain, and severe retinopathy of prematurity. J Pediatr 2013;163:67-72.

18 Hartnett ME, Morrison MA, Smith S, et al. Genetic variants associated with severe retinopathy of prematurity in extremely low birth weight infants. Invest Ophthalmol Vis Sci 2014;55:6194-203.

19 Horbar JD, Carpenter JH, Badger GJ, et al. Mortality and neonatal morbidity among infants 501 to 1500 grams from 2000 to 2009. Pediatrics 2012;129:1019-26.

20 Early Treatment for Retinopathy of Prematurity Cooperative Group. Revised indications for the treatment of retinopathy of prematurity: results of the early treatment for retinopathy of prematurity randomized trial. Arch Ophthalmol 2003;121:1684-94.

21 Chamney S, McGrory L, McCall E, et al. Treatment of retinopathy of prematurity in Northern Ireland, 2000-2011: a population-based study. J AAPOS 2015; 19:223-7.

22 Painter SL, Wilkinson AR, Desai $\mathrm{P}$, et al. Incidence and treatment of retinopathy of prematurity in England between 1990 and 2011: database study. Br J Ophthalmol 2015;99:807-11.

23 Wong HS, Santhakumaran S, Statnikov Y, et al. Retinopathy of prematurity in English neonatal units: a national population-based analysis using NHS operational data. Arch Dis Child Fetal Neonatal Ed 2014;99:F196-202.

24 Isayama T, Lee SK, Mori R, et al. Comparison of mortality and morbidity of very low birth weight infants between Canada and Japan. Pediatrics 2012;130:e957-65.

25 Cryotherapy for Retinopathy of Prematurity Cooperative Group. Multicenter trial of cryotherapy for retinopathy of prematurity. Preliminary results. Arch Ophthalmol 1988;106:471-9.

26 Darlow BA, Elder MJ, Horwood LJ, et al. Does observer bias contribute to variations in the rate of retinopathy of prematurity between centres? Clin Exp Ophthalmol 2008:36:43-6.

27 Phelps DL. It's plus disease, isn't it? Arch Ophthalmol 2007;125:963-4.

28 Kemper AR, Prosser LA, Wade KC, et al. A comparison of strategies for retinopathy of prematurity detection. Pediatrics 2016;137:e20152256.

29 Horbar JD. The Vermont Oxford Network: evidence-based quality improvement for neonatology. Pediatrics 1999;103(Suppl E):350-9.

30 Askie LM, Brocklehurst P, Darlow BA, et al. NeOProM: Neonatal oxygenation prospective meta-analysis collaboration study protocol. BMC Pediatr 2011;11:6.

31 Saugstad OD, Aune D. Optimal oxygenation of extremely low birth weight infants: a meta-analysis and systematic review of the oxygen saturation target studies. Neonatology 2014;105:55-63.

32 Holmström G, Hellström A, Jakobsson $P$, et al. Five years of treatment for retinopathy of prematurity in Sweden: results from SWEDROP, a national quality register. Br J Ophthalmol 2016;100:1656-61. 